\title{
Fate of residual ureteral stump in patients undergoing robot-assisted radical nephroureterectomy for high-risk upper tract urothelial carcinoma
}

\author{
Ram A. Pathak, Ashok K. Hemal \\ Wake Forest University Baptist Medical Center, 1 Medical Center BLVD, Winston-Salem, NC 27157, USA \\ Contributions: (I) Conception and design: All authors; (II) Administrative support: All authors; (III) Provision of study materials or patients: All \\ authors; (IV) Collection and assembly of data: All authors; (V) Data analysis and interpretation: All authors; (VI) Manuscript writing: All authors; (VII) \\ Final approval of manuscript: All authors. \\ Correspondence to: Ashok K. Hemal, MD. Wake Forest University Baptist Medical Center, Department of Urology, 1 Medical Center BLVD, \\ Winston-Salem, NC 27157, USA. Email: ahemal@wakehealth.edu.
}

\begin{abstract}
Background: Management of the distal ureter in radical nephroureterectomy and bladder cuff excision (RNUBCE) is paramount, directly influencing oncologic outcomes. Herein, we analyze the natural history of patients who have undergone robotic radical nephroureterectomy without formal bladder cuff excision and retained ureteral stump and compare this cohort with patients undergoing formal RNUBCE for highrisk upper tract urothelial carcinoma (UTUC).
\end{abstract}

Methods: From February 2008 to October 2018, all patients who underwent robotic RNUBCE by multiple surgeons in a single institution for high-risk UTUC were reviewed. Preoperative, perioperative, and postoperative variables were investigated. Overall survival, cancer specific survival, local recurrencefree survival, distant recurrence-free survival, and bladder recurrence-free survival were compared between the two cohorts. Further management treatments were explored for patients with retained ureteral stump. Follow-up consisted of abdominopelvic/chest imaging and cystoscopy at regular intervals.

Results: A total of 105 patients underwent robotic RNU during the above time period. Of patients with documented 6-month follow-up, approximately $6.6 \%$ of patients had retained ureteral stump. Median follow-up for the entire cohort was 31.5 months with a range of 6 to 114.2 months. Factors that precluded formal BCE were densely, fibrotic reaction near the ureterovesical junction due to prior vascular or pelvic surgery in 5 patients, severe pyonephrosis and continued anesthetic risk in one patient, and surgeon choice (patient co-morbidities) in another patient. Three patients died with metastatic disease and one patient succumbed to cardiovascular compromise. Two additional patients developed local recurrence only at the level of the ureteral stump, with one patient undergoing eventual distal excision, contralateral RNUBCE and radical cystectomy.

Conclusions: In these cases, responsibilities assumed by the surgeon demand the utmost in judgement and skill; however, at times, circumstances prevail such as patient factors and nature/biology of the disease. These factors may prevent adequate excision the complete ureter, ureterovesical junction, and bladder cuff at the time of RNU. In this robotic cohort of patients undergoing RNUBCE for UTUC, not excising the most distal part of the ureter directly translates to inferior oncologic outcomes. Complete ureteral excision with bladder cuff should be performed where possible as this is an integral part of the radical nephroureterectomy. Also, if feasible, adjunctive chemotherapy/immunotherapy treatments should be considered.

Keywords: Radical nephroureterectomy; laparoscopic; robotic; bladder cuff excision; upper tract urothelial carcinoma (UTUC); lymphadenectomy

Submitted Aug 05, 2019. Accepted for publication Sep 04, 2019.

doi: $10.21037 /$ tau.2019.09.14

View this article at: http://dx.doi.org/10.21037/tau.2019.09.14 


\section{Introduction}

Urothelial carcinoma of the upper tract (UTUC) is a relatively uncommon genitourinary malignancy with 3,930 estimated new cases in 2019 (1). According to the Surveillance, Epidemiology, and End Results (SEER) database, 5 -year overall survival can range from $<20 \%$ to $75 \%$ (2). Patients who exhibit high grade on ureteroscopy/ cytology, hydronephrosis, tumor size $>2 \mathrm{~cm}$, invasive features, and multifocal disease are deemed high-risk (3-5). The gold standard treatment for patients with highrisk features is radical nephroureterectomy and bladder cuff excision (RNUBCE).

Critical to disease management is successful excision of the bladder cuff. In fact, SEER data has shown a 1.25to 1.45 -fold greater disease specific mortality in pT3-4 or pT(any) N1-3 patients with incomplete bladder cuff excision (6). When the bladder cuff is taken, management of the distal ureter can be performed in a multitude number of ways including transvesical, extravesical, endoscopic (transurethral) or open surgery. When comparing techniques, endoscopic management has been previously shown to negatively influence recurrence-free survival $(7,8)$. Although many series suggest improved perioperative outcomes utilizing the laparoscopic (9) or robotic approach (10) as compared to open surgery, oncologic outcomes remain controversial $(11,12)$.

Recently, a large systematic review was performed to provide guideline consensus statements on approach of RNUBCE with perioperative and oncologic outcomes data (13). After synthesis of 42 studies including over 7,000 patients, the authors concluded comparable outcomes with the exception of patients undergoing laparoscopic bladder cuff excision who exhibited inferior oncologic outcomes.

Therefore, adequate management of the distal ureter directly translates to oncologic success. Incomplete or poor technique when performing BCE impacts cancer recurrence rates. We aim to examine the natural history of retained ureteral stump and its impact on survival and recurrence rates in a large cohort of patients undergoing robot-assisted RNUBCE.

\section{Methods}

A retrospective review of patients undergoing RNUBCE by multiple surgeons over 10 years [2008-2018] was queried using an institutional review board-approved internal database. Patients were further classified according to surgical approach including open, laparoscopic and robotassisted, yielding a total of 105 patients undergoing robotassisted RNUBCE. Sociodemographic, preoperative, intraoperative, perioperative, pathologic, and follow-up data were recorded for all patients.

\section{Surgical intervention, follow-up and comparison}

Patients who exhibited high risk UTUC were worked up appropriately with ureteroscopic, histopathologic, cytologic, and/or radiographic studies. Port placement was modified through the years with adoption of the newer robotic platforms (Standard $\left.{ }^{\circledR}, \mathrm{S}^{\circledR}, \mathrm{Si}^{\circledR}, \mathrm{Xi}^{\circledR}\right)(14-16)$. Our surgical technique has previously been reported and after January 2009, cases were performed exclusively without intraoperative repositioning (17). Mitomycin C (MMC) was incorporated in our practice regimen in 2012 (18) but was held in all cases without formal bladder cuff excision. Regional lymphadenectomy was performed in patients with high-grade disease in template fashion dependent on the site and side or primary tumor. The operative dictation report was investigated to ensure bladder cuff excision was performed and notated if not.

Follow-up regimen was in accordance to NCCN guidelines and included regular cystoscopy every 3 months for at least 1 year and abdominopelvic and chest imaging at least annually (5). Complications were graded using the modified Clavien classification system at 30- and 90-days postoperative. As part of a multidisciplinary clinic, most patients were seen by medical oncology and decision to proceed with neoadjuvant or adjuvant therapy was obtained on a case-by-case basis. Patient symptomatology and comorbidity status influenced this decision-making process.

\section{Statistical analysis}

Analysis of sociodemographic, preoperative, perioperative, postoperative and long-term follow-up data was performed. OS, CSS, local recurrence (LR), distant recurrence (DR), and bladder recurrence-free survival (BRFS) were calculated using Kaplan-Meier curves with estimated survival rates at 12,36 , and 60 months.

\section{Results}

A total of 105 patients underwent robot-assisted RNUBCE for the indication of high-risk UTUC (Table 1). Operative and pathologic data can be reviewed for patients not 
Table 1 Demographic information for patients undergoing radical nephroureterectomy with and without bladder cuff excision

\begin{tabular}{lcc}
\hline Variables & RNU + BCE $(n=98)$ & RNU - BCE $(n=7)$ \\
\hline Age & 69.9 & 76.7 \\
BMI & 26.9 & 30.36 \\
ASA & 2.95 & 3.14 \\
Gender (F:M) & $30: 68$ & $3: 4$ \\
Race (Caucasian) & $92.8 \%$ & $85.7 \%$ \\
Diabetes & $30.6 \%$ & $42.9 \%$ \\
Heart disease & $32.6 \%$ & $28.6 \%$ \\
COPD & $16.3 \%$ & $14.3 \%$ \\
HTN & $61.2 \%$ & $71.4 \%$ \\
HLD & $58.2 \%$ & $28.6 \%$ \\
Tobacco abuse & $77.5 \%$ & $57.1 \%$ \\
\hline
\end{tabular}

Table 1 depicts patients undergoing radical nephroureterectomy for high-risk upper tract urothelial cancer. Patients were then stratified with formal bladder cuff excision vs. no bladder cuff excision. BMI, body mass index; ASA, American Society of Anesthesiologists score; COPD, chronic obstructive pulmonary disease ; HTN, hypertension; HLD, hyperlipidemia.

undergoing formal bladder cuff excision in Table 2.

Average operating room time was 232.3 minutes (incision time to closure) while estimated blood loss was $125 \mathrm{~mL}$. Average length of stay was 3.14 days with age, medical co-morbidities, and distance to the hospital being significant contributing factors for a majority of patients. $71.4 \%$ of patients had pT2 disease or greater on final pathology with a mean tumor size of $4.8 \mathrm{~cm}$. Clavien $\geq 3$ complications occurred in 1 patient who suffered from cardiovascular collapse secondary to myocardial infarction. One patient received neoadjuvant chemotherapy and 2 patients received adjuvant chemotherapy (Gem/Cis).

Reason for failure of bladder cuff excision and postoperative course is described on Table 3. Common reasons for failure to perform BCE included dense, fibrotic reaction in the pelvis secondary to prior abdominopelvic surgery ( 5 patients) and patient co-morbidity status ( 2 patients). 4 deaths were reported at a follow-up of 29.6 months. When compared to patients who underwent formal bladder cuff excision, overall survival, cancer specific survival, local recurrence-free survival, and distant recurrence-free survival were significantly inferior (Table 4).

\section{Discussion}

High-risk UTUC is best managed by radical nephroureterectomy and complete bladder cuff excision.
A variety of techniques have been developed for distal ureterectomy and BCE including open excision, endoscopic resection (19), incision (20), or un-roofing (21) and concomitant "pluck" technique, and use of LigaSure device (22) or GIA stapler (23). Regardless of approach, bladder cuff excision and management of the distal ureter is critical and can directly influence oncologic outcomes, particularly recurrence rates (24). Mismanagement of the distal ureter can lead to ureteral stump recurrence rates of up to $58 \%$ (25). In more contemporary series, a lack of transvesical bladder cuff excision was noted to have statistically significant poorer cancer-specific survival and non-intravesical recurrence free survival rates (26). When examining transvesical, extravesical and endoscopic techniques for bladder cuff excision, the endoscopic approach was associated with a higher rate of intravesical recurrence (8). In one randomized control trial comparing pure laparoscopic versus open RNUBCE, poorer oncologic outcomes were associated with laparoscopic RNUBCE in patients with high grade or $\geq$ pT3 disease (9).

In our series of 105 patients, we found 7 patients who underwent incomplete bladder cuff excision. Comparative analysis of this cohort revealed inferior oncologic outcomes with respect to overall survival, cancer-specific survival, local recurrence-free survival and distant recurrence-free survival in patients who did not have an excision of bladder cuff. Therefore, surgical success in these patients lies in 
Table 2 Perioperative variables for those patients who did not undergo formal bladder cuff excision

\begin{tabular}{|c|c|}
\hline Perioperative variables & RNU - BCE $(n=7)$ \\
\hline Side & Right: $85.7 \%$; left: $14.3 \%$ \\
\hline Urine cytology & Negative: $28.6 \%$; atypical cells of us: $28.6 \%$; positive: $42.9 \%$ \\
\hline OR time (incision timeout - closing timeout) & 232.3 \\
\hline LOS & 3.14 days \\
\hline pT (operative pathology) & $\mathrm{T}_{0}: 14.3 \%{ }^{*} ; \mathrm{T}_{\mathrm{a}}: 14.3 \% ; \mathrm{T}_{1}: 0 \% ; \mathrm{T}_{2}: 42.9 \% ; \mathrm{T}_{3}: 28.6 \% ; \mathrm{T}_{4}: 0 \%$ \\
\hline High grade & $100 \%$ \\
\hline Tumor size & $4.8 \mathrm{~cm}$ \\
\hline LVI & $14.3 \%$ \\
\hline PSM & $14.3 \%$ \\
\hline LND performed & $57.1 \%$ \\
\hline $\mathrm{LN}+$ & $0 \%$ \\
\hline 90-day complications Clavien $\geq 3$ & $14.3 \%$ \\
\hline
\end{tabular}

*, patient with prior history of endoscopic management with ablation of a mid-ureteral tumor and subsequent development of stricture and chronic hydronephrosis. Follow-up biopsy confirmed high-grade disease and patient elected nephroureterectomy. Final pathology showed no residual carcinoma. RCE, radical nephroureterectomy; BCE, bladder cuff excision; OR, operating room; EBL, estimated blood loss; LOS, length of stay; LVI, lymphovascular invasion; PSM, positive surgical margin; LND, lymph node dissection; LN, lymph node positivity.

Table 3 Patient-specific outcomes after radical nephroureterectomy without bladder cuff excision

\begin{tabular}{|c|c|c|}
\hline Patient & Why bladder cuff excision (BCE) was not performed & Fate \\
\hline 2 & $\begin{array}{l}\text { Dense fibrotic reaction in the pelvis secondary to prior pelvic } \\
\text { surgeries }\end{array}$ & $\begin{array}{l}\text { Death: locally recurrent (at nephrectomy bed) and metastatic } \\
\text { disease }\end{array}$ \\
\hline 3 & Small bowel was plastered in the pelvis & Death: metastatic disease \\
\hline 5 & Dense, fibrotic reaction in pelvis & No evidence of recurrence \\
\hline 6 & $\begin{array}{l}\text { Pyonephrosis with densely, adherent tissue; anesthetic risk; } \\
\text { patient age over } 90 \text { years }\end{array}$ & Intravesical recurrence + recurrence at ureteral stump \\
\hline 7 & $\begin{array}{l}\text { Dense, fibrotic reaction in pelvis secondary to prior } \\
\text { abdominopelvic surgery }\end{array}$ & $\begin{array}{l}\text { Intravesical and contralateral recurrence. Patient underwent distal } \\
\text { ureterectomy, contralateral nephroureterectomy and radical cystectomy }\end{array}$ \\
\hline
\end{tabular}


Table 4 Comparison of patients undergoing radical nephroureterectomy (RNU) and formal bladder cuff excision (BCE) with those with retained ureteral stump

\begin{tabular}{lll}
\hline Oncologic outcomes & P value & $\mathrm{HR}$ and $95 \% \mathrm{Cl}$ \\
\hline Overall survival & 0.0009 & $0.19(0.07-0.51)$ \\
Cancer-specific survival & 0.0001 & $0.09(0.02-0.30)$ \\
Local recurrence-free survival & 0.0033 & $0.16(0.05-0.54)$ \\
Distant recurrence-free survival & 0.0004 & $0.17(0.06-0.45)$ \\
Bladder recurrence-free survival & 0.14 & $0.31(0.07-1.46)$ \\
\hline
\end{tabular}

efficient management of the distal ureter.

Oncologic outcomes after robot-assisted RNUBCE have previously been investigated (10). Overall-survival and cancer-specific survival at 2 and 5 years after robotassisted RNUBCE has been estimated at $86.9 \%$ and $62.6 \%$ (OS) and $92.9 \%$ and $69.5 \%$ (CSS), respectively (27). Thus, the robotic approach has been proven to be efficacious in disease management. As previously stated, pure laparoscopy has been associated with worse oncologic outcomes in patients with high grade or pT3/4 disease (9). The drawback to the pure laparoscopic approach lies within the bladder cuff excision and reconstruction portion of the procedure. With the advancement of robotic-assistance, bladder reconstruction has become more technically feasible.

Reasons for incomplete bladder cuff excision were investigated by examining the operative note. The leading majority included a dense, fibrotic reaction in the pelvis secondary to multiple prior pelvic surgeries in approximately $60 \%$ of patients. One patient was deemed an anesthetic risk, intraoperatively while plastering of the small bowel and dense ureteral fibrosis (presumed secondary to multiple prior ureteroscopies) was the culprit in the remaining 3 patients. Prior abdominopelvic surgery did affect a majority of these patients but should not be the sole focus of this analysis as roughly $32 \%$ of patients with complete BCE also had history of prior surgery. At times, intraoperative circumstances may dictate a change in surgical strategy and certain patient factors may impede the surgical success.

When longitudinally following these 7 patients, 5 developed recurrence with 3 patients succumbing to metastatic disease and eventual death. The other death in this cohort was secondary to cardiovascular collapse within 30 days of the operation. One patient developed recurrence at the ureteral stump, bladder recurrence and contralateral ureteral recurrence and underwent successful ipsilateral ureteral excision (pTaN0), contralateral radical nephroureterectomy [pTaN0 (high grade)] and radical cystectomy [pTaNo (high grade)]. Adjuvant treatments were used sparingly in this cohort (2 of 7 patients) likely secondary to development of chronic kidney disease.

Therefore, failure to perform BCE can directly translate to poorer oncologic outcomes. Every attempt should be made to perform an oncologically sound surgery to include formal excision of bladder cuff regardless of approach open or minimally-invasive. In our series of 105 patients, the robotic approach was successfully able to manage the distal ureter in over $93 \%$ of cases.

\section{Conclusions}

When assessing oncologic outcomes of UTUC, bladder cuff excision is critical to the success of the surgery. In this institutional cohort with multiple surgeons, the robotic approach can yield efficient management of the distal ureter in a majority of cases. At times, patient-related factors may preclude initial attempts at bladder cuff excision; however, every effort should be taken to perform an oncologically sound surgery.

\section{Acknowledgments}

Funding: None.

\section{Footnote}

Provenance and Peer Review: This article was commissioned by the Guest Editor (Ashok K. Hemal) for the series "Robotic-assisted Urologic Surgery" published in Translational Andrology and Urology. The article was sent for external peer review organized by the Guest Editor and the editorial office. 
Conflicts of Interest: All authors have completed the ICMJE uniform disclosure form (available at http://dx.doi. org/10.21037/tau.2019.09.14). The series "Robotic-assisted Urologic Surgery" was commissioned by the editorial office without any funding or sponsorship. AKH served as the unpaid Guest Editor of the series and serves as the unpaid editorial board member of Translational Andrology and Urology from May 2019 to Apr 2021. RAP has no other conflicts of interest to declare.

Ethical Statement: The authors are accountable for all aspects of the work in ensuring that questions related to the accuracy or integrity of any part of the work are appropriately investigated and resolved. The study did not require ethics approval given the retrospective analysis of previously collected data.

Open Access Statement: This is an Open Access article distributed in accordance with the Creative Commons Attribution-NonCommercial-NoDerivs 4.0 International License (CC BY-NC-ND 4.0), which permits the noncommercial replication and distribution of the article with the strict proviso that no changes or edits are made and the original work is properly cited (including links to both the formal publication through the relevant DOI and the license). See: https://creativecommons.org/licenses/by-nc-nd/4.0/.

\section{References}

1. Siegel RL, Miller KD, Jemal A. Cancer Statistics, 2019. CA Cancer J Clin 2019;69:7-34.

2. Raman JD, Messer J, Sielatycki J, et al. Incidence and survival of patients with carcinoma of the ureter and renal pelvis in the USA, 1973-2005. BJU International 2011;107:1059-64.

3. Rouprêt M, Colin P, Yates D. A new proposal to risk stratify urothelial carcinomas of the upper urinary tract (UTUCs) in a pre-definitive treatment setting: low risk versus high risk UTUCs. Eur Urol 2014;66:181-3.

4. Rouprêt M, Babjuk M, Comperat E, et al. European Association of Guidelines on Upper Urinary Tract Urothelial Carcinoma: 2017 Update Eur Urol 2018;73:111-22.

5. National Comprehensive Cancer Network. Bladder Cancer Version 4. 2019. Available online: https://www. nccn.org/professionals/physician_gls/pdf/bladder.pdf

6. Lughezzani G, Sun M, Perrotte P, et al. Should bladder cuff excision remain the standard of care at nephroureterectomy in patients with urothelial carcinoma of the renal pelvis? A Population-based study. Eur Urol 2010;57:956-62.

7. Kapoor A, Dason S, Allard CB et al. The impact of method of distal ureter management during radical nephroureterectomy on tumor recurrence. Can Urol Assoc J 2014;8:E845-52.

8. Xylinas E, Rink M, Cha EK, et al. Impact of distal ureter management on oncologic outcomes following radical nephroureterectomy for upper tract urothelial carcinoma. Eur Urol 2014;65:210-7.

9. Simone G, Papalia R, Guaglianone S, et al. Laparoscopic versus open nephroureterectomy: perioperative and oncologic outcomes from a randomized prospective study. Eur Urol 2009;56:520-6.

10. Pathak RA, Hemal AK. Techniques and Outcomes of Robot-assisted Nephro-ureterectomy for Upper Tract Urothelial Carcinoma. Eur Urol Focus 2018;4:657-61.

11. Stewart GD, Humphries K, Cutress M, et al. Longterm comparative outcomes of open versus laparoscopic nephroureterectomy for upper urinary tract urothelialcell carcinoma after a median follow-up of 13 years. J Endourol 2011;25:1329.

12. Ni S, Tao W, Chen Q, et al. Laparoscopic versus open nephroureterectomy for the treatment of upper urinary tract urothelial carcionma: a systematic review and cumulative analysis of comparative studies. Eur Urol 2012;61:1142-53.

13. Peyronnet B, Seisen T, Dominguez-Escrig J, et al. Oncologic outcomes of laparscopic nephroureterectomy versus open radical nephroureterectomy for upper tract urothelial carcionma: An european association of urology guidelines systematic review. Eur Urol Focus 2019;5:205-23.

14. Pathak RA, Patel M, Hemal AK. Comprehensive Approach to Port Placement Templates for Robotiassisted Laparoscopic Urologic Surgeries. J Endourol 2017;31:1269-76.

15. Patel MN, Aboumohamed A, Hemal AK. Does transition from the da Vinci Si to Xi robotic platform impact single-docking technique for robot-assisted laparoscopic nephroureterectomy. BJU Int 2015;116:990-4.

16. Patel MN, Hemal AK. Does advancing technology improve outcomes? Comparison of Da Vinci Standard/ $\mathrm{S} / \mathrm{Si}$ to the Xi robotic platforms during robotic nephroureterectomy. J Endourol 2018;32:133-8.

17. Hemal AK, Stansel I, Babbar P, et al. Robotic-assisted nephroureterectomy and bladder cuff excision without 
intraoperative repositioning. Urology 2011;78:357-64.

18. O’Brien T, Ray E, Singh R, et al. Prevention of bladder tumors after nephroureterectomy for primary upper urinary tract urothelial carcinoma: a prospective, multicenter, randomized clinical trial of a single postoperative intravesical dose of Mitomycin C (the ODMIT-C Trial). Eur Urol 2011;60:703-10.

19. Palou J, Caparros J, Orsola B, et al. Transurethral resection of the intramural ureter as the first step of nephroureterectomy. J Urol 1995;154:43-4.

20. Kurzer E, Leveillee R, Bird V, et al. Combining hand assisted laparoscopic nephroureterectomy with cystoscopic circumferential excision of the distal ureter without primary closure of the bladder cuff - is it safe? J Urol 2006;175:63-7.

21. Shalhav AL, Elbahnasy A, McDougall E, et al. Laparoscopic nephroureterectomy for upper tract transitional-cell cancer: technical aspects. J Endourol 1998;12:345-53.

22. Tsivian A, Benjamin S, Sidi A, et al. A sealed laparoscopic nephroureterectomy: a new technique. Eur Urol 2007;52:1015-9.

23. Kerbl K, Clayman R, McDougall E, et al. Laparoscopic nephroureterectomy: evaluation of first clinical series. Eur Urol 1993;23:431-6.

24. Mullen E, Ahmed K, Challacombe B. Systematic Review of open versus laparoscopic versus robot-assisted nephroureterectomy. Rev Urol 2017;19:32-43.

25. Strong DW, Pearse H, Tank E, et al. The ureteral stump after nephroureterectomy. J Urol 1976;115:654-5.

26. Krabbe LM, Westerman M, Bagrodia A, et al. Surgical mangement of the distal ureter during radical nephroureterectomy is an independent predictor of oncologic outcomes: results of a current series and a review of literature. Urol Oncol 2014;32:54.e19-26.

27. Aboumohamed AA, Krane LS, Hemal AK. Oncologic outcomes following robot-assisted laparoscopic nephroureterectomy with bladder cuff excision for upper tract urothelial carcinoma. J Urol 2015;194:1561-6.
Cite this article as: Pathak RA, Hemal AK. Fate of residual ureteral stump in patients undergoing robot-assisted radical nephroureterectomy for high-risk upper tract urothelial carcinoma. Transl Androl Urol 2020;9(2):856-862. doi: 10.21037/tau.2019.09.14 